\title{
Electro-Chemical Induced Fusion OR - The Case of the Missing Neutrons
}

Once again the physics world has been shaken by a discovery which suggests that our conventional wisdom has a big hole in it. Shaken too by the fact it should hear of it first through the newspapers and not through a scientific journal after passing through all the traditional processes of refereeing although these are not necessarily very perceptive when the ideas and the phenomena are against all experience.

Towards the end of March, worried by the growing flood of rumour and false reports, Martin Fleischmann of the University of Southampton, UK, and Stan Pons of the University of Utah, USA, decided to report publicly at a press conference on their electrochemical experiments in Utah that had been going on for five years. To their astonishment, Fleischmann maintains, the story became banner headlines in countries all over the world and dozens of laboratories are now repeating the experiments and contemplating new ones. Some are also recalling a peculiar explosion in an electro-chemical cell containing lithium that was never satisfactorily explained.

\section{The Apparatus}

The apparatus used by Fleischmann and Pons consisted essentially of a Dewar flask filled with heavy water laced with LiOD in which was inserted a central palladium cathode $(1-4 \mathrm{~mm}$ diameter by $10 \mathrm{~cm}$ long, machined from a cast rod), and an annular anode comprised of platinum wire wound helically on a glass frame. Various temperature recording devices enabled them to make accurate calorimetric measurements. We are talking of something say $30 \mathrm{~cm}$ high. A row of them, each immersed in a bath of water plus power supplies and all, occupied just the end of an ordinary laboratory.

\section{The Phenomenon}

Put simply, what Fleischmann and Pons found was that after 'charging' a cell for a certain period (a week or so, even months for larger electrodes) by applying a few volts, the heat generated, typically some $50 \mathrm{k} \mathrm{J} / \mathrm{h} \mathrm{cm} \mathrm{cm}^{2}$, rose to a value that was twice the power fed in. This state would then continue for a hundred hours and more, perhaps in- definitely. They are confident of their calorimetric measurements and oblique confirmation comes from one of the cells which went dead (probably they think, through poisoning of the cathode by the anode, a well-known phenomenon in electro-chemistry) when the energy balanced to within a $\mathrm{mW}$ or so compared to an input of around $1 \mathrm{~W}$.

\section{The Supposition}

The excess energy cannot come from chemical reactions. We are assured by the chemists that there just is not that amount of chemical energy available even if one could release it. This suggested a nuclear source and indeed, it was with a view to seeing if deuterons could be persuaded to cold fuse that the experiments were first designed.

Without being dogmatic, Fleischmann and Pons suggest a mechanism based on the following reactions:

$$
\begin{aligned}
D_{2} O+e^{-} & \rightleftharpoons D_{\text {adsorbed }}+O^{-} \\
D_{\text {ads }} & \rightleftharpoons D_{\text {lattice }} \\
D_{\text {ads }}+D_{2} O+e^{-} & \rightleftharpoons D_{2}+O D^{-} \\
D_{\text {ads }}+D_{\text {ads }} & \rightleftharpoons D_{2}
\end{aligned}
$$

On applying a voltage to the cell, deuterons cross the electrode surface and diffuse into the octahedral holes of the expanded face centred cubic lattice. It is this diffusion process that takes so long. Fleischmann points out that electrochemically it is easy to change the chemical potential by $0.8 \mathrm{eV}$ whereas translated into pressure $(R T / 2) \log p / p_{0}$ would imply $p / p_{0}=10^{27}$ (a figure not attainable as the internal rupture pressure of palladium is around $4000 \mathrm{~atm}$ ).

Fleischmann suspects that in some holes, clusters of at least four deuterons collect and that fusion occurs in these. $\mathrm{He}$ also argues that the Coulomb repulsion in these holes could be highly anisotropic.

The normal signatures of DD fusion - tritium and neutrons have been detected - not directly the $2.45 \mathrm{MeV}$ neutrons produced in the DD $\rightarrow{ }^{3} \mathrm{He}+\mathrm{n}$ reaction, but $2.2 \mathrm{MeV} \gamma$ resulting from $\mathrm{np}$ reactions in the surrounding tank. The tritium, presumably from DD $\rightarrow p+T$ has been measured by bremsstrahlung counting of $\beta$ decays.

In a typical case, the number of neutron reactions registered is three times background (six per hour total), but the energy discrimination is good. Separate integrated dosimeter measurements put the source flux at $4 \times 10^{4} \mathrm{n} / \mathrm{s}$ while the tritium counts would point to some $10^{4} \mathrm{DD}$ fusions/s. As the two reaction channels (at high energy) have comparable cross-sections, the figures are reasonably consistent.

\section{The Mystery}

BUT, to produce the amount of heat determined by the calorimetric measurements would require a production rate $10^{9}$ times that unless the principal channel is perfectly clean fusion to ${ }^{4} \mathrm{He}$ and we are seeing only evidence from side reactions. In a two-body collision, momentum considerations rule out $D+D \rightarrow$ ${ }^{4} \mathrm{He}$, but in a multi-body interaction perhaps momentum sharing of the $24 \mathrm{MeV}$ released is a possibility. Or might it be the $\mathrm{D}+{ }^{6} \mathrm{Li} \rightarrow 2 \times{ }^{4} \mathrm{He}$ reaction that is the main source.

There can be little doubt that the discrepancy between the calorimetry and nuclear measurements is real and the explanation that they are just wrong is the least probable. It is a pity that comparable experiments with hydrogen have not yet been done nor definitive measurements made on $\mathrm{He}$ production, but they are in the pipeline.

Fleischmann and Pons believe it to be a problem for the nuclear physicists to identify the interaction channel which swallows all those neutrons at birth and they look forward to supervising experiments on DTO. But no doubt physicists from other fields will also want to have their say. Certainly the solid state people will want to do sums on the energy stored in the lattice: the hydrogen-palladium system has some very odd characteristics - see, for example, Norskov J.K., Hydrogen - metal Interaction, Europhys. News 19 (1988) 65 and the Li-Pd system is far from understood.

Whatever the outcome, there is going to be a lot of excitement in the field in the months to come and whether the table top fusion reactor is just round the corner or not, there is still a fascinating lot of physics to sort out.

E.N. Shaw 